\title{
Phytol Induces Apoptosis and ROS-Mediated Protective Autophagy in Human Gastric Adenocarcinoma AGS Cells
}

\author{
YeonWoo Song ${ }^{1}$ and Somi Kim $\mathrm{Cho}^{2 *}$
}

${ }^{1}$ Faculty of Biotechnology, College of Applied Life Sciences, SARI, Jeju National University, Jeju 690-756, Republic of Korea

${ }^{2}$ Subtropical Horticulture Research Institute, Jeju National University, Jeju 690-756, Republic of Korea

\begin{abstract}
Phytol, a diterpene alcohol produced from chlorophyll, is used widely as a food additive and an aromatic ingredient. However, the molecular mechanisms behind the cytotoxic effects of phytol in cancer cells are not understood. The current study demonstrated that phytol induces apoptosis in human gastric adenocarcinoma AGS cells, as evidenced by an increased cell population in the sub-G1 phase, downregulation of Bcl-2, upregulation of Bax, the activation of caspase- 9 and -3 , PARP cleavage, and depolarization of the mitochondrial membrane. In addition, phytol induced autophagy, as evidenced by the induction of acidic vesicle accumulation, the conversion of microtubule-associated protein LC3-I to LC3-II, and the suppression of Akt, mTOR, and p70S6K phosphorylation. Most importantly, pretreatment with chloroquine, a lysosomal inhibitor, strongly augmented phytol-induced apoptosis in AGS cells, suggesting that phytol could induce protective autophagy. Furthermore, co-treatment with a reactive oxygen species (ROS) scavenger enhanced the phytol-induced cytotoxicity, which is accompanied by the decrease in the levels of p62 as well as the percentage of acidic vesicular organelles (AVOs) positive cells, suggesting that the cytoprotective Nrf2 pathway was induced by phytol. Taken together, these findings provide valuable insights into the mechanisms by which phytol induces cell death in AGS cells.
\end{abstract}

Keywords: AGS human gastric adenocarcinoma cells; Apoptosis; Autophagy; Phytol; ROS

\section{Introduction}

Phytol (3,7,11,15-tetramethyl-2-hexadecen-1-ol) is an acyclic monounsaturated diterpene alcohol from chlorophyll. It is an aromatic ingredient used in many fragrant compounds, and is also found in some cosmetic and non-cosmetic products [1]. Known for its medicinal value, phytol has antinociceptive and antioxidant activities [2], as well as anti-inflammatory [3] and immunostimulatory effects [4]. Phytol also elicits antimicrobial activity against Mycobacterium tuberculosis [5,6] and Staphylococcus aureus [7]. Several reports have assessed the anticancer activities of plant extracts that contain phytol as a major component [8-10]. Similar to these findings, a recent reports assessed the apoptosis induction in hepatocellular carcinoma cells [11] and the cytotoxic effects of phytol using MTT assays in several tumor cell lines in vitro [12]. However, the molecular mechanism by which phytol induced cell death remains unclear. In this study, we explored the molecular mechanisms by which phytol induces cell death in AGS gastric cancer cells.

Cell death plays an important role in cancer growth and progression, as well as the efficacy of chemotherapy. Apoptosis is thought to be the principal mechanism by which anti-cancer drugs kill cells. Apoptosis is a highly conserved form of cell suicide that is regulated by programmed cellular signaling pathways that control tissue homeostasis and/or eliminate damaged or infected cells [13]. There are two major representative apoptotic pathways: the extrinsic pathway, which is mediated by death receptors (DRs), and the intrinsic pathway, which is mediated by mitochondria. The death-receptormediated pathway activates caspase- 8 by forming the death-induced signaling complex (DISC) [14]. Activated caspase-8 then cleaves Bid into truncated Bid (tBid), which connects the death receptor pathway to the mitochondrial pathway by translocating from the cytosol to the mitochondria. The mitochondrial pathway is initiated by the loss of mitochondrial membrane potential (MMP, $\Delta \Psi \mathrm{m}$ ) and release of cytochrome $c$, which activates caspase- 9 and its downstream effector caspases that cleave different substrates to eventually lead to dismantling of the cell $[15,16]$
Autophagy is an evolutionarily conserved cellular degradation pathway for long-lived proteins and organelles [17]. Induction of autophagy might maintain cancer survival under metabolic stress and mediate resistance to anticancer therapies [18]. Thus inhibition of autophagy enhances cancer cell death and potentiates various anticancer therapies $[19,20]$. High levels of autophagy can induce a form of cell death known as type II cell death or autophagic cell death [21]. From a molecular point of view, several cellular signaling pathways play a role in regulating autophagy, including phosphatidylinositol 3-kinase (PI3K)/Akt/mammalian target of rapamycin (mTOR). Previous studies have shown that inhibiting Akt and its downstream target mTOR contribute to the initiation of autophagy [22]. During the initial stages of autophagy cellular proteins, organelles, and the cytoplasm are sequestered and engulfed by intracellular double membrane-bound structures called autophagosomes. The microtubule-associated protein 1 light chain 3 (LC3), a mammalian homolog of the yeast Atg8, a cytosolic form of LC3 (LC3-I) is conjugated to phosphatidylethanolamine to form LC3-II, which is recruited to autophagosomal membranes [23]. These autophagosomes mature by fusing with lysosomes to form the autolysosomes, in which the sequestered proteins and organelles are digested by lysosomal hydrolases and recycled to sustain cellular metabolism [24]. Through a comprehensive analysis of LC3-binding, p62 was identified as an LC3-interacting protein [25-27]. Because LC3II present in the inner membrane of the autophagosome is degraded together with other cellular constituents by lysosomal proteases, p62

*Corresponding author: Somi Kim Cho, Faculty of Biotechnology, Jeju National University, 66 Jejudaehakno, Jeju, 690-756, Korea; Tel: +82-64-754-3348; Fax: +82-64-756-3351; E-mail: somikim@jejunu.ac.kr, phd.kim.somi@gmail.com

Received: June 23, 2015; Accepted: September 07, 2015; Published September 09, 2015

Citation: Song Y, Cho SK (2015) Phytol Induces Apoptosis and ROS-Mediated Protective Autophagy in Human Gastric Adenocarcinoma AGS Cells. Biochem Ana Biochem 4: 211. doi:10.4172/2161-1009.1000211

Copyright: @ 2015 Song Y, et al. This is an open-access article distributed under the terms of the Creative Commons Attribution License, which permits unrestricted use, distribution, and reproduction in any medium, provided the original author and source are credited. 
trapped by LC3 is transported selectively into the autophagosome. The p62 protein localizes to sites of autophagosome formation and can associate with both the LC3 and ubiquitinated proteins [26]. Therefore, p62 is considered to act as a receptor for ubiquitinated proteins, which it sequesters into the autophagosome [28] and the impaired autophagy is accompanied by accumulation of $\mathrm{p} 62$.

Reactive oxygen species (ROS) is a term that describes the collective species formed by the incomplete reduction of oxygen, including superoxide anion $(\mathrm{O} 2-)$, hydrogen peroxide $\left(\mathrm{H}_{2} \mathrm{O}_{2}\right)$, and hydroxyl radicals (HO•) [29]. Intracellular redox homeostasis is a key determinant of cell fate: excessive ROS production usually results in cytotoxic effects and might lead to apoptotic cell death, whereas moderate levels of ROS function as second messengers to regulate diverse cellular processes such as cell survival, proliferation, and metastasis [30,31]. The accumulation of ROS can induce autophagy, which in turn facilitates the clearance of excessive ROS to protect cells from oxidative damage; this might reflect the balance of either cell survival or death [32,33]. During oxidative stress, ROS-mediated KEAP1 inhibition results in the activation of Nuclear factor-erythroid 2-related factor $2(\mathrm{Nrf} 2)$ and the expression of its target genes $\mathrm{p} 62$, heme oxygenase-1 (HO-1) and $\mathrm{NAD}(\mathrm{P}) \mathrm{H}$ dehydrogenase, quinine 1 (NQO1) [34]. Moreover, p62 can interact directly with KEAP1 to promote the non-canonical activation of $\mathrm{Nrf} 2$ and creating a positive feedback loop that further amplifies p62 expression [35]. Therefore, p62 stimulates autophagy and the Nrf2-mediated antioxidant response during oxidative stress.

The main objective of this study was to examine the cytotoxic mechanism of action of phytol, an aromatic ingredient used in many fragrant compounds. Here, we demonstrate that phytol induces apoptosis and autophagy in human gastric adenocarcinoma AGS cells. Phytol-induced apoptosis was enhanced by inhibiting autophagy or suppressing ROS accumulation. These findings strongly suggest that phytol-induced autophagy might function as a survival pathway against apoptosis, and that the combination treatment with phytol and an autophagy inhibitor or ROS scavenger might exert promising therapeutic effects in stomach cancer.

\section{Materials and Methods}

\section{Cell lines and cell culture}

AGS human gastric adenocarcinoma cells and human dermal fibroblast cells were obtained from the ATCC (Manassas, VA, USA). AGS cells were cultured in F-12K medium supplemented with $10 \%$ heat-inactivated fetal bovine serum (FBS) and $1 \%$ antibiotics at $37^{\circ} \mathrm{C}$ in a humidified atmosphere containing 5\% CO2.

\section{Reagents}

F-12K medium, trypsin/EDTA, and fetal bovine serum (FBS) were purchased from Invitrogen (Carlsbad, CA, USA). Phytol (Figure 1A), Hoechst 33342 dye, acridine orange dye (AO), 3-(4,5-dimethylthiazol2-yl)-2,5-diphenyltetrazolium bromide (MTT), propidium iodide (PI), chloroquine (CQ), RNase A, 2 $\square, 7 \square$-dichlorofluorescin diacetate (H2DCF-DA), and anti-LC3 and -actin antibodies were purchased from Sigma Chemical Co. (St. Louis, MO, USA). The BDTM Mitoscreen (JC-1) kit was purchased from BD Biosciences (Franklin Lakes, NJ, USA). Anti-caspase-9, -caspase-3, -PARP, -Bcl-2, -Akt, -p-Akt, -mTOR, -p-mTOR, - p70S6K, -p-p70S6K, and -Bax antibodies were purchased from Cell Signaling Technology (Danvers, MA, USA). Western blotting polyvinylidene fluoride (PVDF) membranes were purchased from Merck Millipore (Darmstadt, Germany).
(A)
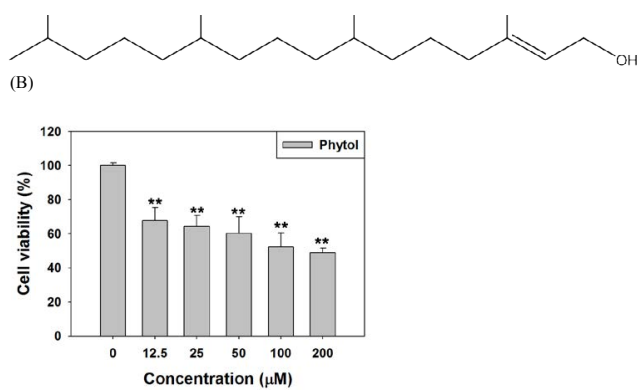

Figure 1: (A) Chemical structure of phytol (3,7,11,15-tetramethylhexadec-2-en1-ol). (B) The viability of cells treated with various concentrations of phytol for 24 $h$. The results shown are the means \pm S.Ds. of three independent experiments $\left({ }^{* *} p<0.01\right.$ vs. control)

\section{Cell viability assays}

The effects of the treatments on cell viability were determined using MTT assays, which are based on the conversion of MTT to formazan crystals by mitochondrial dehydrogenases. Briefly, cells were plated in 96-well plates at an initial density of $6 \times 103$ cells/well. After a 24-h incubation, the cells were treated with phytol alone or combination of $60 \mu \mathrm{M}$ chloriquine or $2 \mathrm{mM} \mathrm{NAC}$ and then incubated for an additional $24 \mathrm{~h}$. At the indicated time points $20 \mu \mathrm{L}$ of MTT solution $(5 \mathrm{mg} / \mathrm{mL})$ was added to each well, and the plates were incubated in a humidified environment for 3-4 h. The supernatant was then removed, and the crystals were dissolved in 150- $\square$ L DMSO. The absorbance at a wavelength of $570 \mathrm{~nm}$ was measured in the microplate reader (Tecan, Grodig, Austria).

\section{Cell morphology}

AGS cells were placed in $60-\mathrm{mm}$ plates at a density of $3 \times 104$ cells/ $\mathrm{mL}$, and then treated with phytol after $24 \mathrm{~h}$. After an additional $24 \mathrm{~h}$, $10 \square \mathrm{M}$ Hoechst or AO was added to each well, and the plates were incubated for $10 \mathrm{~min}$ at $37^{\circ} \mathrm{C}$. The stained cells were then observed under a fluorescence microscope (Olympus, Tokyo, Japan).

\section{Flow cytometry}

To detect the sub-diploid population cells were detached from plates by the addition of trypsin-EDTA, washed in phosphate-buffered saline (PBS), fixed in 70\% ethanol, treated with RNase A (25 ng/ $\mathrm{mL}$ ), and finally stained with $40 \mu \mathrm{g} / \mathrm{mL}$ PI. The generation of ROS was measured using H2DCF-DA as a substrate. Cells were incubated with $10 \mu \mathrm{M}$ H2DCF-DA for $30 \mathrm{~min}$, harvested, resuspended in PBS, and analyzed. To detect autophagy cells were stained with $10 \mu \mathrm{M} \mathrm{AO}$, harvested, and suspended in PBS. The data from 10,000 cells/sample were analyzed using CellQuest software (Becton Dickson, Franklin Lakes, NJ, USA). Each experiment was repeated at least three times.

\section{Western blotting analysis}

Cell lysates were prepared by incubating cells in $150 \mu \mathrm{L}$ of lysis buffer (100 mM Tris-HCl, pH 8, $250 \mathrm{mM} \mathrm{NaCl}, 0.5 \%$ Nonidet P-40, $1 \times$ protease inhibitor cocktail), disrupted by sonication, and extracted at $4 \square \mathrm{C}$ for $30 \mathrm{~min}$. The protein concentration was determined using BCATM protein assays (Pierce, Rockford, IL, USA). Aliquots of the lysates were separated using $12-15 \%$ SDS-PAGE and transferred to PVDF membranes using a glycine transfer buffer (192 mM glycine, 25 $\mathrm{mM}$ Tris- $\mathrm{HCl} \mathrm{pH} \mathrm{8.8,} \mathrm{and} 20 \%$ methanol (v/v). After blocking with 
$5 \%$ non-fat dried milk, the membrane was incubated for $2 \mathrm{~h}$ with primary antibodies followed by $30 \mathrm{~min}$ with secondary antibodies in Tris-buffered saline (TBS) containing $0.5 \%$ Tween-20. Most primary antibodies were used in 1:1,000 dilutions except for $\beta$-actin $(1: 10,000$ dilution), and the horseradish peroxidase-conjugated goat anti-rabbit IgG secondary antibodies (Vector Laboratories, Burlingame, CA, USA) were used in 1:5,000 dilutions. Protein bands were detected using the BS ECL-Plus Kit (Biosesang, Gyeonggi-do, Korea).

\section{Reverse transcription-polymerase chain reaction (RT-PCR)}

Total RNA was extracted from cells using TRIzol reagent (Invitrogen). Reverse transcription was carried out using the reverse transcription system (Promega, Madison, WI, USA). Polymerase chain

(A)

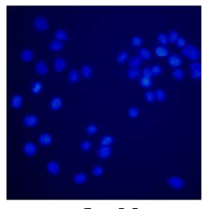

$0 \mu \mathrm{M}$

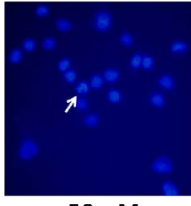

$50 \mu \mathrm{M}$

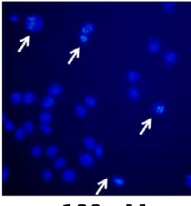

$100 \mu \mathrm{M}$

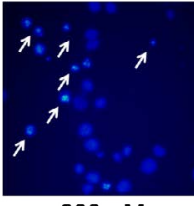

$200 \mu \mathrm{M}$
(B)

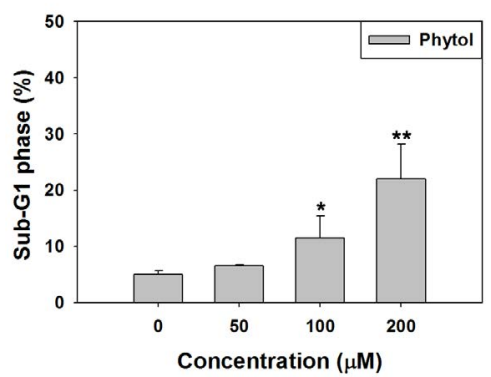

(C)

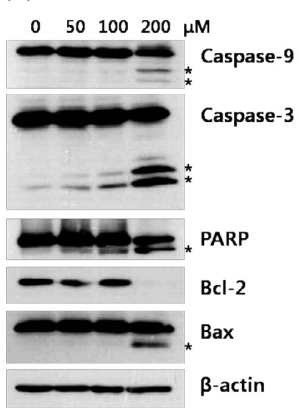

(D)

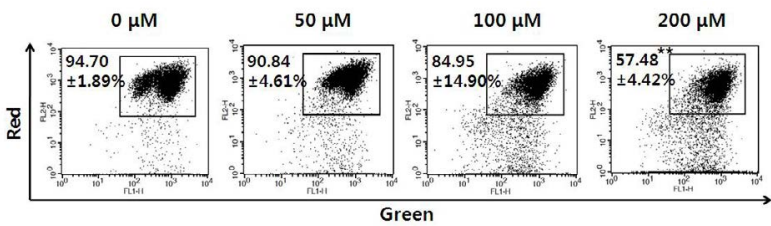

Figure 2: Phytol induces apoptosis in AGS cells. (A) Cells were treated with the indicated concentrations of phytol for $24 \mathrm{~h}$, stained with Hoechst 33342, and observed under a fluorescent microscope. (B) AGS cells were treated for $24 \mathrm{~h}$ and the DNA content was analyzed using flow cytometry. Statistically significant differences are presented as ${ }^{*} p<0.05$ and ${ }^{* *} p<0.01$. (C) Lysates were prepared from cells treated with DMSO or phytol for $24 \mathrm{~h}$, and western blotting was performed using caspase-9, caspase-3, PARP, Bcl-2, and Bax antibodies; (*) indicates the cleaved form of the protein. (D) Mitochondrial membrane potential, as assessed using $\mathrm{JC}-1$ staining. ${ }^{* *} p<0.01$. reaction (PCR) was performed using Taq polymerase (Enzynomics, Daejeon, Korea) with the following cycling conditions: initiation at $95^{\circ} \mathrm{C}$ for $1 \mathrm{~min}$, followed by 35 cycles of 40 -s denaturation at $95^{\circ} \mathrm{C}$, 40 -s annealing at $57^{\circ} \mathrm{C}$, and 40 -s elongation at $72^{\circ} \mathrm{C}$. PCR products $(5$ $\mu \mathrm{L}$ ) were separated on $1.2 \%$ agarose gels and then stained with ethidium bromide. PCR primers for amplification were as follows: p62, forward 5'-AAGCCGGGTGGGAATGTTG-3', reverse 5'-GCTTGGCCCTTCGGATTCT-3'; HO-1, forward 5'-ACTTCCCAGAAGAGCTGCAC-3', reverse 5'-GCTTGAACTTGGTGGCACTG-3'; NQO-1, forward 5'-GGGCAAGTCCATCCCAACTG-3', reverse 5'-GCAAGTCAGGGAAGCCTGGA-3'; GAPDH, forward 5'-GAGAAGGCTGGGGCTCATTT-3', reverse 5'-AGTGATGGCATGGACTGTGG-3'

\section{Statistical analysis}

The significance of differences between groups was determined using one-way analysis of variance (ANOVA). The western blots shown are representative of a group of experiments, and graphs represent the means \pm standard deviations of at least two independent experiments.

\section{Results}

\section{Phytol induces apoptosis in AGS cells}

The antiproliferative effects of phytol on AGS cells are shown in Figure 1B. Phytol significantly inhibited the growth of AGS cells compared to untreated control. To determine whether the reduced cell viability of cells was attributable to apoptosis, we assessed morphological changes by staining cell nuclei with Hoechst 33342 . Phytol induced the fragmentation and condensation of AGS cell nuclei in a concentrationdependent manner (Figure 2A). Next, the effects of phytol on the cell cycle were analyzed using flow cytometry. The percentage of cells in the sub-G1 peak is shown in Figure 2B. There was a significant and dosedependent increase in the sub-G1 fraction from $5.02 \%$ in control cells to $22.03 \%$ in the cells treated with $200 \mu \mathrm{M}$ phytol. The results of western blotting revealed that phytol decreased the levels of the anti-apoptotic protein Bcl-2 and increased the cleaved form of caspase-9, -3, PARP, and Bax (Figure 2C). It was suggested that chemical-induced apoptosis is often, but not always, associated with a loss of the mitochondrial membrane potential $(\Delta \Psi \mathrm{m})$ that is caused by increased leakiness of the inner mitochondrial membrane [36,37]. The non-toxic fluorescent probe JC-1 becomes concentrated in mitochondria as red fluorescent aggregates when the $\Delta \Psi \mathrm{m}$ is high, and is converted to green monomers when the $\Delta \Psi \mathrm{m}$ is lost. As shown in Figure 2D, phytol increased the number of mitochondria with disrupted membrane potential in a dosedependent manner. These results suggest that phytol induced apoptotic cell death by causing DNA damage and mitochondrial membrane dysfunction.

\section{Phytol induces autophagy in AGS cells}

Acridine orange (AO) staining was used to analyze the formation of acidic vesicular organelles (AVOs) or autophagolysosome vacuoles, which occurs as the result of fusion between autophagosomes and lysosomes and is a key feature of autophagy [38]. Large numbers of AVOs (red aggregates) were detected in phytol-treated AGS cells, indicating an induction of autophagy (Figure 3A). The conversion of LC3-I into LC3-II followed by the localization and aggregation of LC3-II in autophagosomes is an autophagosomal marker [39]. Previous studies confirmed that inhibition of the Akt/mTOR pathway triggers autophagy [19]. Activated Akt, which provides key signals to downstream molecules such as mTOR, can inhibit autophagy and promote cell survival [40]. As shown in Figure 3B, phytol suppressed the phosphorylation of Akt, mTOR, and p70S6K, 
Citation: Song Y, Cho SK (2015) Phytol Induces Apoptosis and ROS-Mediated Protective Autophagy in Human Gastric Adenocarcinoma AGS Cells. Biochem Anal Biochem 4: 211. doi:10.4172/2161-1009.1000211

Page 4 of 7

(A)
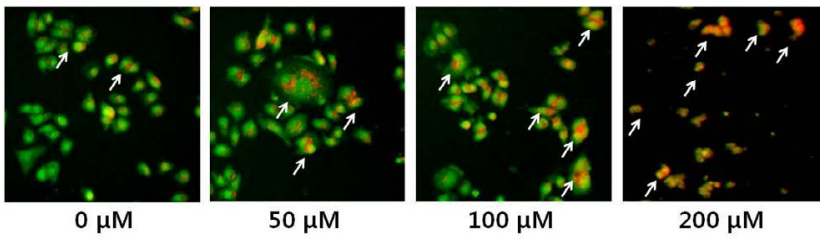

(B)

$0 \quad 50 \quad 100200 \mu \mathrm{M}$

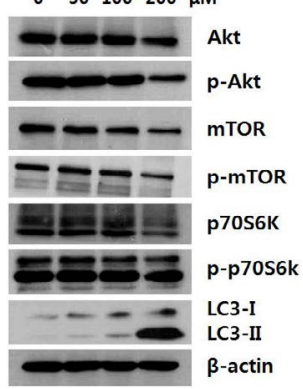

(C)

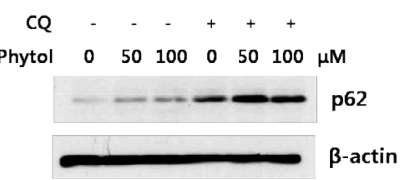

(D)

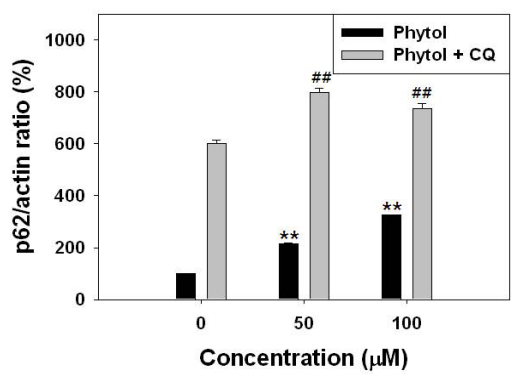

Figure 3: Detection of autophagy in AGS cells treated with phytol for 24 h. (A) Acidic vesicular organelles (AVOs) were examined by incubating the cells with $10 \mu \mathrm{M}$ acridine orange (AO), and the images were analyzed using a fluorescence microscope. (B) Western blotting for Akt, p-Akt, mTOR, p-mTOR, p70S6K, p-p70S6K, LC3, and $\beta$-actin after the treatment of cells with the indicated concentrations of phytol for $24 \mathrm{~h}$. (C) AGS cells were treated with phytol in the absence or presence of $60 \mu \mathrm{M}$ chloroquine (CQ). Cells were then harvested and analyzed by western blotting using the indicated antibodies. (D) The band intensity of the blots shown in (C) was quantified using the ImageJ software. Data were normalized to the amount of $\beta$-actin in each sample. ( $" p<0.01$ vs. control, \#\#<0.01 vs. CQ treated cells).

and increased the conversion of LC3-I to LC3-II. Because p62 is localized to autophagosomes via an interaction with LC3 and is subsequently degraded by the autophagy-lysosome system, treatment of a lysosomotropic agent can lead to a marked accumulation of p62 [41]. The levels of p62 were greater in the cells treated with both phytol and chloroquine (CQ), a lysosomotropic agent, than those in the cells treated with phytol alone, indicating that phytol activated the autophagic response (Figure $3 \mathrm{C}$ and $3 \mathrm{D}$ ).

\section{Phytol induces protective autophagy in AGS cells}

Because the role of autophagy in the fate of cancer cells remains controversial, we next investigated whether phytol-induced autophagy functioned as a pro-survival or pro-death mechanism. To determine the biological role of autophagy in phytol-mediated apoptotic cell death, CQ was used to disrupt lysosomal function and prevent completion of the autophagic flux. As shown in Figure 4A, the number of apoptotic bodies was increased when cells were co-treated with phytol and CQ compared with phytol alone. In addition, the sub-G1 population was increased two-fold by co-treatment with phytol and CQ compared with those treated with phytol alone (Figure $4 \mathrm{~B}$ ). Furthermore, the amount of the cleaved forms of caspase- 3 and PARP proteins was also increased

(A)

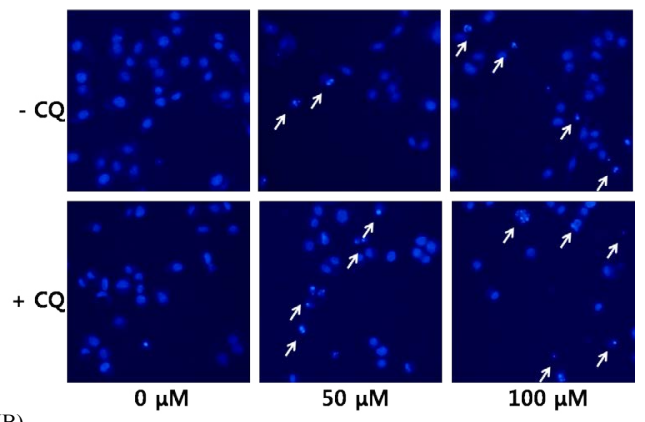

(B)

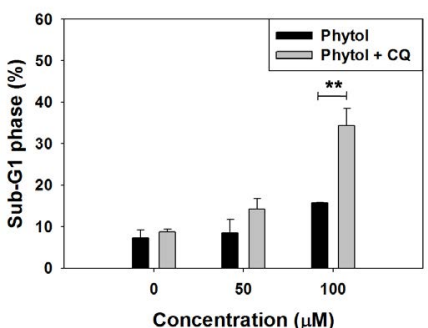

(C)

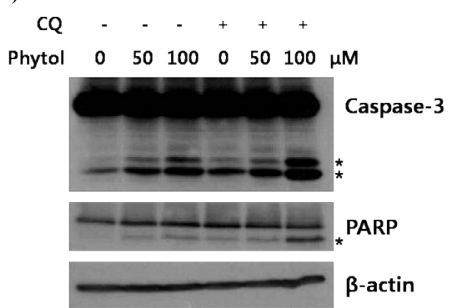

(D)

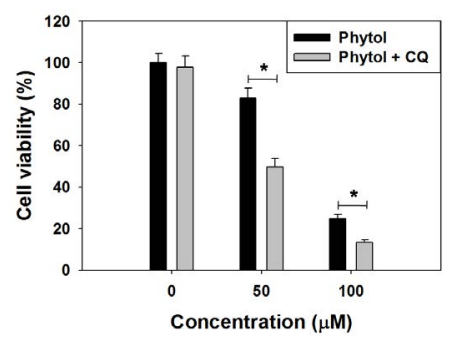

Figure 4: Phytol induces protective autophagy in AGS cells. Cells were treated with phytol alone or together with $60 \mu \mathrm{M}$ chloroquine (CQ) for 24 h. (A) Apoptotic cells were analyzed using Hoechst staining. (B) AGS cells were treated as indicated for $24 \mathrm{~h}$ and DNA content was analyzed using flow cytometry. Results are presented as the means \pm S.Ds. of three independent experiments. "* $p<0.01$. (C) Western blotting was performed using caspase- 3 and PARP antibodies; ( $\left(^{*}\right)$ indicates the cleaved form of the protein. (D) Cell viability was determined using MTT assays. ${ }^{*} p<0.05$. 


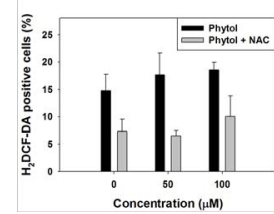

(B)

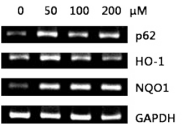

(C)

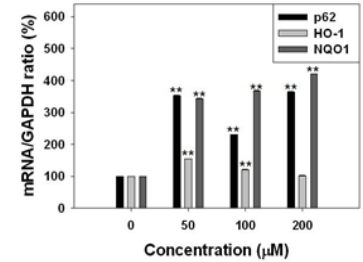

(D) NAC - . + + +

Phytol $0 \quad 50100 \quad 0 \quad 50 \quad 100 \quad \mu M$

$-1-0-102$
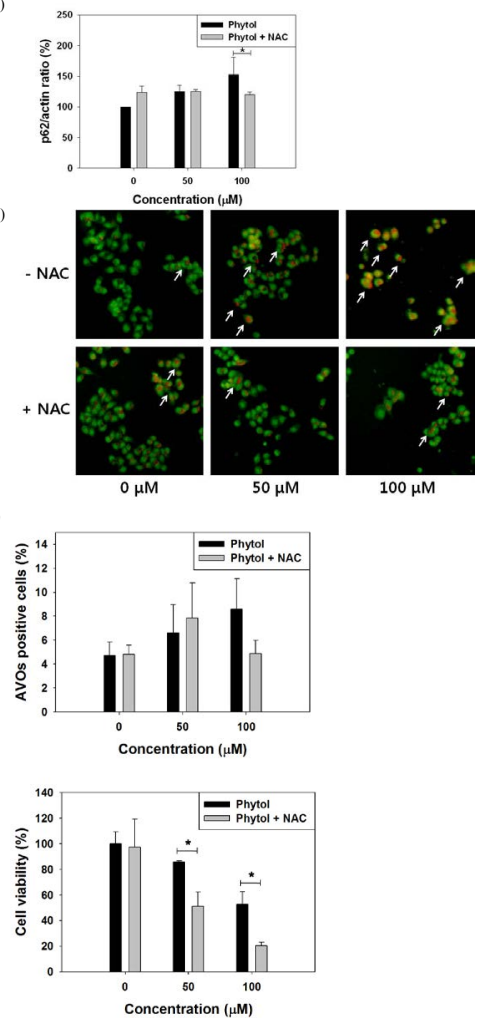

Figure 5: Phytol induces ROS-dependent cytoprotective pathways in AGS cells. (A) AGS cells were treated with phytol in the absence or presence of $2 \mathrm{mM}$ NAC for $24 \mathrm{~h}$, and then stained with $\mathrm{H}_{2}$ DCF-DA. The $\mathrm{H}_{2}$ DCF-DA fluorescence intensity was measured using flow cytometry. (B) The mRNA levels of Nrf2 target genes was assessed after treatment with phytol for $24 \mathrm{~h}$. Total RNA was harvested from AGS cells, reverse transcribed, and amplified using gene-specific primers. (C) The values were normalized to the amount of each mRNA in untreated AGS cells. ${ }^{* *} p<0.01$. (D) AGS cells were treated with phytol in the absence or presence of $2 \mathrm{mM} \mathrm{NAC}$ for $24 \mathrm{~h}$, and p62 protein levels were measured using western blotting. (E) The intensities of the bands shown in (D) were quantified using the ImageJ software. ${ }^{*} p<0.05$. Acidic vesicular organelles (AVOs) were analyzed using fluorescent microscopy $(F)$ or flow cytometry $(G)$ in AGS cells treated with phytol and/or NAC for $24 \mathrm{~h}$. (H) The viability of AGS cells was determined using MTT assay. ${ }^{*} p<0.05$.

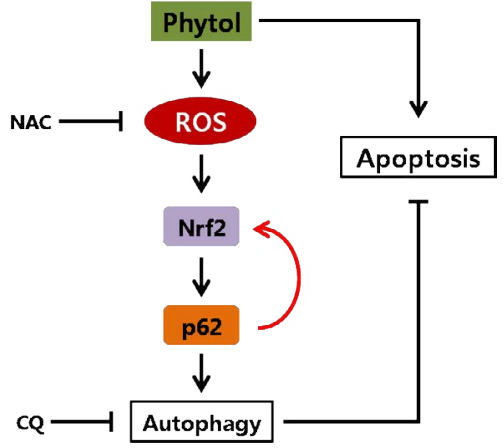

Figure 6: A schematic diagram summarizing the cytotoxic effects of phytol in human gastric adenocarcinoma AGS cells. Phytol increases ROS levels, which results in the induction of autophagy. ROS-induced Nrf2 enhances the expression of the antioxidant genes and $p 62$. In turn, p62 protein associates with Keap1 (Jain et al., and Komatsu et al.) and activates Nrf2, completing a positive feedback loop (red arrow). Increased p62 levels can induce autophagy in AGS cells, and also has a protective role against phytol-induced apoptosis. Therefore, the induction of apoptosis by phytol treatment can be enhanced by targeting ROS generation or inhibiting the protective autophagy. ROS, Reactive oxygen species; Nrf2, Nuclear factor-erythroid 2-related factor 2; NAC, N-acetylL-cysteine; CQ, Chloroquine.

after co-treatment with phytol and CQ (Figure 4C). The results of MTT assays showed that cell viability was decreased further when autophagy was blocked using CQ (Figure 4D). Taken together, these data strongly suggest that inhibiting autophagy enhanced apoptotic cell death in AGS cells treated with phytol.

\section{Phytol induces ROS mediated Nfr2 cytoprotective pathways}

Because the results described above showed that phytol increased the levels of p62 (Figure 3C) and it has been reported p62 promotes ROS production [41], we speculated that phytol might activate the Nrf2 cytoprotective pathway. To investigate this possibility, we first examined ROS levels in AGS cells by measuring the percentage of H2DCF-DA-positive cells, which is proportional to ROS production. As shown in Figure 5A, treatment with 50 and $100 \mu \mathrm{M}$ phytol increased the percentage of H2DCF-DA-positive cells by $17.65 \%$ and $18.58 \%$, respectively, compared with control (14.81\%). Interestingly, the expressions of the Nrf2 target genes, p62, HO-1, and NQO1 were increased dramatically, in contrast to the slight increase in ROS production (Figure 5B and $5 \mathrm{C}$ ). In order to confirm that all these responses are due to the increase in ROS production, the cells were treated with the ROS scavenger $\mathrm{N}$-acetyl-L-cysteine (NAC). The percentage of phytol-induced H2DCF-DA-positive cells was reduced in the presence of NAC (Figure 5A) and correspondingly p62 protein levels as well as the percentage of AVOs positive cells were reduced (Figure 5D-5G). In particular, co-treatment with NAC enhanced the cytotoxic effects of phytol (Figure $5 \mathrm{H}$ ). Together, these results suggest that phytol-induced ROS can facilitate protective autophagy via Nrf2 antioxidant pathway (Figure 6).

\section{Discussion}

Phytol is widespread in nature because it is a constituent of chlorophyll [42]. It is a common food additive, and information regarding the oral bioavailability of phytol in mice revealed that it is well absorbed [43]. Moreover, comprehensive toxicological data are available. For example, the acute oral LD50 of phytol in rats was reported to be $>10,000 \mathrm{mg} / \mathrm{kg}$, and it was not mutagenic [1]. Some reports suggested that phytol derivatives might activate nuclear hormone receptors and modulate gene expression and cell differentiation [44]. 
Phytol can activate peroxisome proliferator-activated receptor a (PPARa) directly and thereby regulate the expression of genes related to lipid metabolism [45]. It also exerts therapeutic effects in models of arthritis and seizures in mice $[46,47]$. In addition, several reports have revealed that plant extracts containing phytol as a major component have anticancer activities [8-10].

Our laboratory has been interested in phytol since we identified that Citrus unshiu leaf extract, which contains phytol as a major component, induces autophagic cell death in human gastric adenocarcinoma AGS cells (to be published in Journal of the Korean Society for Applied Biological Chemistry, 2015). While we were investigating the cytotoxic mechanism of action of phytol, one research group reported that phytol induced concentration-dependent cytotoxic responses in seven cell lines (MCF-7, HeLa, HT-29, A-549, Hs294T, MDA-MB-231, and PC-3 cells) as evaluated using the MTT assays that revealed IC50 values ranging from 8.79 to $77.85 \mu \mathrm{M}$ [12]. However, in the current studies milder toxicity was detected against human gastric adenocarcinoma AGS cells at the concentrations used (IC50 $147.67 \pm 5.63 \mu \mathrm{M}$ ). This suggests that phytol might induce protective pathways in AGS to render cells resistant to cell death. Therefore, these results emphasize the importance of understanding the molecular mechanisms behind the effects of phytol to enhance its therapeutic efficacy.

The current data demonstrated that phytol inhibited proliferation and induced both apoptosis and autophagy in AGS cells. Apoptosis and autophagy have many common regulators, and crosstalk between these pathways regulates cell fate in response to cellular stress. The complex interaction between apoptotic and autophagic pathways necessitates the careful consideration of both pathways to understand cell death phenomena fully [48]. Previous studies revealed that several anticancer drugs induced both apoptosis and autophagy in cancer cells [49]. The relationship between autophagy and apoptosis is complex, and varies according to the individual cell types and the specific stress placed upon the cell [50]. Although it remains controversial whether autophagy kills cancer cells or sustains their survival under conditions of stress, an increasing number of reports have provided data to support the hypothesis that autophagy promotes the survival of cancer after chemotherapy or radiation therapy, thereby allowing cancer cells to escape drug-induced apoptosis [51,52]. CQ is a lysomotropic drug that alters lysosomal $\mathrm{pH}$, prevent autophagosome-lysosome fusion. By blocking the last step of the autophagy pathway, leads to the accumulation of ineffective autophagosomes and cell death [53]. The current results revealed that the combination of CQ and phytol inhibited proliferation and increased apoptosis significantly in AGS cells, as evidenced by the cleavage of caspase- 3 and PARP. These results suggest that phytol-induced autophagy protects against apoptosis.

The KEAP1-Nrf2 system is a major cellular defense mechanism against oxidative stress [54]. Several reports have revealed that during oxidative stress, ROS-mediated KEAP1 inhibition results in the activation of Nrf2 and the expression of its target gene p62 [34]. In addition, p62 can interact with KEAP1 via its KIR domain to promote the non-canonical activation of Nrf2, thereby creating a positive feedback loop that further amplifies p62 expression [35]. In particular, p62 has a positive effect on autophagy and the Nrf2-mediated antioxidant response during conditions of oxidative stress. Furthermore, p62 is the highest specific adaptor protein for autophagy and plays a central role in clearance of protein aggregates $[28,35]$. Considering that p62 is a receptor for ubiquitinated proteins and organelle to degrade them into the lysosomes, the current results showed that phytol induces ROS production and increases p62 expression and then the Nrf2-p62 loop may function to keep the level of p62 protein. Consistent with this, scavenging ROS by co-treatment with NAC reduced the levels of p62 and acidic vesicular organelles (AVOs) as well as overall cell viability.

Several stimuli that induce ROS production can also induce autophagy, including nutrient starvation, hypoxia, oxidative stress, and some chemotherapeutic agents [55]. Alterations in ROS levels and autophagy play a crucial role in the initiation and progression of cancer, and both are recognized as potential targets for cancer treatment $[56,57]$. Major sources of cellular ROS are the mitochondrial electron transport chain, the NADPH oxidase (NOX) complex, and the endoplasmic reticulum $[29,30]$. A recent report revealed that gambogic acid, a polyprenylated xanthone, could promote apoptosis and autophagy in colorectal cancer cells, and that inhibiting autophagy enhanced the sensitivity to gambogic acid treatment. In addition, the accumulation of intracellular ROS via 5-lipoxygenase (5-LOX) was required for gambogic acid-induced autophagy [10]. Similarly, the current results showed that phytol-induced autophagy was prevented when ROS generation was blocked by treatment with NAC. Further studies to identify the markers responsible for ROS generation after treatment with phytol are required to elucidate the precise connection between ROS and autophagy.

In summary, the current study revealed that phytol could induce both apoptosis and protective autophagy in AGS cells. The autophagy inhibitor CQ enhanced the inhibitory effects of phytol on cell proliferation and promoted phytol-induced apoptosis in gastric cancer cells. These effects were regulated by ROS generation, which could lead to protective autophagy via a Nrf2-p62 feedback loop. These data suggest that there is crosstalk between apoptosis and ROS-induced autophagy in phytol-treated AGS cells, and provide novel insights into the treatment of cancer using phytol. The effects of phytol could be enhanced by combination treatment with either autophagy inhibitors or ROS scavengers.

\section{Acknowledgements}

This research was supported by Basic Science Research Program through the National Research Foundation of Korea (NRF), funded by the Ministry of Education (2013R1A1A2A10012017).

\section{References}

1. McGinty D, Letizia CS, Api AM (2010) Fragrance material review on phytol Food Chem Toxicol 48 Suppl 3: 59-63.

2. Santos CC, Salvadori MS, Mota VG, Costa LM, de Almeida AA et al. (2013) Antinociceptive and Antioxidant Activities of Phytol In Vivo and In Vitro Models. Neurosci J 2013: 949452

3. Ryu KR, Choi JY, Chung S, Kim DH (2011) Anti-scratching behavioral effect of the essential oil and phytol isolated from Artemisia principles Pamp. in mice. Planta Med 77: 22-26.

4. Lim SY, Meyer M, Kjonaas, RA, Ghosh SK (2006) Phytol-based novel adjuvants in vaccine formulation: 1 . assessment of safety and efficacy during stimulation of humoral and cell-mediated immune responses. J. Immune Based Ther. Vaccines $4: 6$.

5. Rajab MS, Cantrell CL, Franzblau SG, Fischer NH (1998) Antimycobacteria activity of (E)-phytol and derivatives: a preliminary structure-activity study. Planta Med 64: 2-4.

6. Saikia D, Parihar S, Chanda D, Ojha S, Kumar JK, et al. (2010) Antitubercular potential of some semisynthetic analogues of phytol. Bioorg Med Chem Lett 20: $508-512$

7. Inoue Y, Hada T, Shiraishi A, Hirose K, Hamashima H, et al. (2005) Biphasic effects of geranylgeraniol, teprenone, and phytol on the growth of Staphylococcus aureus. Antimicrob Agents Chemother 49: 1770-1774.

8. Hsu HF, Huang KH, Lu KJ, Chiou SJ, Yen JH, et al. (2011) Typhonium blume extract inhibits proliferation of human lung adenocarcinoma A549 cells via 
Citation: Song Y, Cho SK (2015) Phytol Induces Apoptosis and ROS-Mediated Protective Autophagy in Human Gastric Adenocarcinoma AGS Cells. Biochem Anal Biochem 4: 211. doi:10.4172/2161-1009.1000211

induction of cell cycle arrest and apoptosis. J Ethnopharmacol 135: 492-500.

9. Hsu HF, Wu YC, Chang CC, Houng JY (2011) Apoptotic effects of bioactive fraction isolated from Glossogynetenufolia on A549 human lung cancer cells. J. Taiwan. Inst. Chem. E 4: 556-562.

10. Jiang R, Sun L, Wang Y, Liu J, Liu X, et al. (2014) Chemical composition, and cytotoxic, antioxidant and antibacterial activities of the essential oil from ginseng leaves. Nat. Prod. Commun 9: 865-868

11. Kim CW, Lee HJ, Jung, JH, Kim YH, Jung DB, et al. (2015) Activation of Caspase-9/3 and Inhibition of Epithelial Mesenchymal Transition are Critically Involved in Antitumor Effect of Phytol in Hepatocellular Carcinoma Cells. Phytother. Res 29: 1026-1031.

12. Pejin B, Kojic V, Bogdanovic G (2014) An insight into the cytotoxic activity of phytol at in vitro conditions. Nat Prod Res 28: 2053-2056.

13. Elmore S (2007) Apoptosis: a review of programmed cell death. Toxicol Pathol 35: $495-516$

14. Debatin KM, Krammer PH (2004) Death receptors in chemotherapy and cancer Oncogene 23: 2950-2966.

15. Wolf BB, Green DR (1999) Suicidal tendencies: apoptotic cell death by caspase family proteinases. J Biol Chem 274: 20049-20052.

16. Cory S, Adams JM (2002) The Bcl2 family: regulators of the cellular life-ordeath switch. Nat Rev Cancer 2: 647-656.

17. Park EJ, Choi KS, Kwon TK (2011) $\hat{I}^{2}$-Lapachone-induced reactive oxygen species (ROS) generation mediates autophagic cell death in glioma U87 MG cells. Chem Biol Interact 189: 37-44.

18. Yuan G, Yan SF, Xue H, Zhang P, Sun JT, et al. (2014) Cucurbitacin I induces protective autophagy in glioblastoma in vitro and in vivo. J Biol Chem 289 : 10607-10619.

19. Kim KW, Mutter RW, Cao C, Albert JM, Freeman M, et al. (2006) Autophagy for cancer therapy through inhibition of pro-apoptotic proteins and mammalian target of rapamycin signaling. J Biol Chem 281: 36883-36890.

20. Apel A, Herr I, Schwarz H, Rodemann HP, Mayer A (2008) Blocked autophagy sensitizes resistant carcinoma cells to radiation therapy. Cancer Res 68: 1485-1494.

21. Baehrecke EH (2005) Autophagy: dual roles in life and death? Nat Rev Mol Cell Biol 6: 505-510.

22. Guertin DA, Sabatini DM (2007) Defining the role of mTOR in cancer. Cancer Cell 12: 9-22.

23. Tanida I, Waguri S (2010) Measurement of autophagy in cells and tissues. Methods Mol Biol 648: 193-214.

24. Mizushima N (2004) Methods for monitoring autophagy. Int J Biochem Cell Biol 36: $2491-2502$

25. Komatsu M, Waguri S, Koike M, Sou YS, Ueno T (2007) Homeostatic levels of p62 control cytoplasmic inclusion body formation in autophagy-deficient mice. Cell 1: 1149-1163.

26. Bjørkøy G, Lamark T, Brech A, Outzen H, Perander M, et al. (2005) p62/ SQSTM1 forms protein aggregates degraded by autophagy and has a protective effect on huntingtin-induced cell death. J Cell Biol 171: 603-614

27. Pankiv S, Clausen TH, Lamark T, Brech A, Bruun JA, et al. (2007) p62 SQSTM1 binds directly to Atg8/LC3 to facilitate degradation of ubiquitinated protein aggregates by autophagy. J Biol Chem 282: 24131-24145.

28. Johansen T, Lamark T (2011) Selective autophagy mediated by autophagic adapter proteins. Autophagy 7: 279-296.

29. Chandel NS, Budinger GR (2007) The cellular basis for diverse responses to oxygen. Free Radic Biol Med 42: 165-174.

30. Wu WS (2006) The signaling mechanism of ROS in tumor progression. Cancer Metastasis Rev 25: 695-705.

31. Inokuma T, Haraguchi M, Fujita F, Tajima Y, Kanematsu T (2009) Oxidative stress and tumor progression in colorectal cancer. Hepatogastroenterology 56: 343-347.

32. Shi Y, Tang B, Yu PW, Tang B, Hao YX, et al. (2012) Autophagy protects against oxaliplatin-induced cell death via ER stress and ROS in Caco-2 cells. PLoS One 7: 51076 .

33. Scherz-Shouval R, Elazar Z (2011) Regulation of autophagy by ROS physiology and pathology. Trends Biochem Sci 36: 30-38.
34. Jain A, Lamark T, Sjøttem E, Larsen KB, Awuh JA, et al. (2010) p62/SQSTM1 is a target gene for transcription factor NRF2 and creates a positive feedback loop by inducing antioxidant response element-driven gene transcription. J Bio Chem 285: 22576-22591.

35. Komatsu M, Kurokawa H, Waguri S, Taguchi K, Kobayashi A, et al. (2010) The selective autophagy substrate p62 activates the stress responsive transcription factor Nrf2 through inactivation of Keap1. Nat. Cell Biol 1:213-223.

36. Salvioli S, Ardizzoni A, Franceschi C, Cossarizza A (1997) JC-, but not DiOC6(3) or rhodamine 1 , is a reliable fluorescent probe to assess delta psi changes in intact cells: implication for studies on mitochondrial functionality during apoptosis. FEBS Lett 41: 77-82.

37. Sun XM, MacFarlane M, Zhuang J, Wolf BB, Green DR, et al. (1999) Distinct caspase cascades are initiated in receptor-mediated and chemical-induced apoptosis. J Biol Chem 274: 5053-5060.

38. Kanzawa T, Germano IM, Komata T, Ito H, Kondo Y, et al. (2004) Role of autophagy in temozolomide-induced cytotoxicity for malignant glioma cells. Cell Death Differ 11: 448-457.

39. Kabeya Y, Mizushima N, Ueno T, Yamamoto A, Kirisako T, et al. (2000) LC a mammalian homologue of yeast Apg8p, is localized in autophagosome membranes after processing. EMBO J 19: 5720-5728.

40. Yap TA, Garrett MD, Walton MI, Raynaud F, de Bono JS, et al. (2008) Targeting the PI3K-AKT-mTOR pathway: progress, pitfalls, and promises. Curr Opin Pharmacol 8: 393-412

41. Mathew R, Karp CM, Beaudoin B, Vuong N, Chen G, et al. (2009) Autophagy suppresses tumorigenesis through elimination of p62. Cell 137: 1062-1075.

42. Baxter JH (1968) Absorption of chlorophyll phytol in normal man and in patients with Refsum's disease. J Lipid Res 9: 636-641.

43. Mize CE, Avigan J, Baxter JH, Fales HM, Steinberg D (1966) Metabolism of phytol-U-14C and phytanic acid-U-14C in the rat. J Lipid Res 7: 692-697.

44. Hansen RP (1966) Occurrence of phytanic acid in rumen bacteria. Nature 210: 841.

45. Goto T, Takahashi N, Kato S, Egawa K, Ebisu S, et al. (2005) Phytol directly activates peroxisome proliferator-activated receptor alpha (PPARalpha) and regulates gene expression involved in lipid metabolism in PPARalpha-expressing HepG2 hepatocytes. Biochem. Biophys. Res. Commun 337: 440-445.

46. Hultqvist M, Olofsson P, Gelderman KA, Holmberg J, Holmdahl R (2006) A new arthritis therapy with oxidative burst inducers. PLoS Med 3: 348.

47. Costa JP, Ferreira PB, De Sousa DP, Jordan J, Freitas RM (2012)Anticonvulsant effect of phytol in a pilocarpine model in mice. Neurosci Lett 523: 115-118.

48. Filippi-Chiela EC, Villodre ES, Zamin LL, Lenz G (2011) Autophagy interplay with apoptosis and cell cycle regulation in the growth inhibiting effect of resveratrol in glioma cells. PLoS One 6: e20849.

49. Song KS, Kim JS, Yun EJ, Kim YR, Seo KS, et al. (2008) Rottlerin induces autophagy and apoptotic cell death through a PKC-delta-independent pathway in HT1080 human fibrosarcoma cells: the protective role of autophagy in apoptosis. Autophagy 4: 650-658.

50. Zhang H, Kong X, Kang J, Su J, Li Y, et al. (2009) Oxidative stress induces parallel autophagy and mitochondria dysfunction in human glioma U251 cells. Toxicol Sci 110: 376-388.

51. Fritsche P, Seidler B, Schüler S, Schnieke A, Göttlicher M, et al. (2009) HDAC2 mediates therapeutic resistance of pancreatic cancer cells via the $\mathrm{BH}$-only protein NOXA. Gut 58: 1399-1409.

52. Carew JS, Nawrocki ST, Cleveland JL (2007) Modulating autophagy for therapeutic benefit. Autophagy 3: 464-467

53. Amaravadi RK, Yu D, Lum JJ, Bui T, Christophorou MA, et al. (2007) Autophagy inhibition enhances therapy-induced apoptosis in a Myc-induced model of Iymphoma. J Clin Invest 117: 326-336.

54. Hayes JD, McMahon M (2009) NRF2 and KEAP1 mutations: permanent activation of an adaptive response in cancer. Trends Biochem Sci 34: 176-188.

55. Lee J, Giordano S, Zhang J (2012) Autophagy, mitochondria and oxidative stress: cross-talk and redox signalling. Biochem J 441: 523-540.

56. Mah LY, Ryan KM (2012) Autophagy and cancer. Cold Spring Harb Perspect Biol 4: 008821.

57. Hale AN, Ledbetter DJ, Gawriluk TR, Rucker EB 3rd (2013) Autophagy: regulation and role in development. Autophagy 9: 951-972 